\title{
Design and Implementation of a Portable Name Card Typesetting System
}

\author{
Chih-Horng Ke \\ Department of Information \\ Management, Chang Jung \\ Christian University \\ Taiwan
}

\author{
Chao-Hsien Hsieh \\ Department of Computer \\ Science and Information \\ Engineering, National Cheng \\ Kung University
}

\author{
Chiang Lee \\ Department of Computer \\ Science and Information \\ Engineering, National Cheng \\ Kung University
}

\begin{abstract}
The goal of this study is to develop a real-time personalized digital name card typesetting system with seamless operability across various platforms. The system includes an authentication module, a template selection module, a text typesetting and merger module, a frame selection and merger module, and a QR code generator and merger module. The system authentication module proposes a potential authentication mechanism between server and client. The template selection module entails making a choice of template from the system-integrated gallery. The text typesetting and merger module proposes the methodology of automatic typesetting that is under fixed font and size restrictions. After it is merged with the last picture, the frame selection and merger module combines one of the pictures from the frame gallery. The QR code generator and merger module is essentially a flowchart of how the QR code is generated. A personalized name card is the end product of these modules. The prototype generation shows that the system works well as a name card typesetting system. Finally, the system shows promising potential for applications to the emerging and proliferating market of smart phones.
\end{abstract}

\section{Keywords}

mobile device, name card, QR code, typesetting, smart phone.

\section{INTRODUCTION}

Exchanging name cards is a popular way to get contact information in social and formal gatherings. They provide instantaneous and concise information of a new acquaintance or potential business partner. Personal and company details are easily relayed through this medium. Although rich in information, it is rather easy for name cards to be misplaced or lost. In years past, Rolodex was once a popular way to manage name cards, but for e-generation people it becomes inconvenient because it may not be handy when they need it.

Smart phones are handheld computers integrated with mobile phone interfaces that allow multitasking applications to run on hardware. They combine the functions of a camera phone and a personal digital assistant (PDA). In recent years, as the number of smart phone users has increased dramatically, so have the quantity, quality, and diversity of smart phone applications. The application software recognizes name cards and then stores them into a mobile phone, such as CamCard INTSIG Information Co., Ltd, Milpitas, CA. or WorldCard Mobile PENPOWER Technology Ltd., Hsinchu, Taiwan, R.O.C.
Currently, the disadvantage of the existing mobile software is that it is not able to recognize various types and formats of name cards perfectly and cannot guarantee accuracy. At present, the major types of transmission for mobile phones include Optical Character Recognition (OCR), handwriting, and file forwarding by means of Bluetooth or QR code scanning [1-4]. Each of these techniques has some irreplaceable and nice features. The advantage of OCR is being able to make quick copies. The contribution of using handwriting is to assist memory while going through every name card. The feature of file forwarding is that it is easy to transfer electronic name cards compared to sending files through email or downloading files from data storage. But, the major problem of these electronic name card systems is that they can hardly recognize a card correctly $100 \%$ of the time by current apps such as CamCard and WorldCard Mobile. There is a great need for verification before an electronic card in the mobile phone is transmitted.

Commercially, some name card applications exist, but these applications are not without shortcomings. Some of them are enumerated as follows: (1) Traditional system of name cards cannot be searched for and dialed or emailed at the same time. (2) Currently, incompatibility issues have been a source of error in electronic name cards. (3) Existing application software cannot make, access, exchange, or encode electronic name cards efficiently. (4) Existing name card systems do not provide encryption and decryption functions for added security. (5) Existing mobile software cannot produce a name card that allows for customizable selection of template, frame, size of QR code, and color of QR code.

The goal of this research is to implement a new name card system called "portable name card typesetting system" (PNCTS), which generates a dynamic format for the name card using smart phones. The application features dynamic management and generation of name cards. The challenge is how to seamlessly organize and integrate the name card, QR code, typesetting mechanism, and mobile device. In the event that professionals suddenly run out of physical name cards, an application that can create name cards in real-time is a possible solution. This study proposes the combination of the QR code with name cards due to some main advantages: (1) accuracy of the information that would be scanned and (2) the potential for encoding personal data within the QR code. (3) The size of QR code in PNCTS can be adjusted dynamically. (4) Name card is easy to be generated by several simple steps. (5) To personal option, the layout templates and frames for $\mathrm{QR}$ code can be picked by users including a preferred size and color. (6) To personal option, the color of QR code is not only a black color, but also many other colors. This proposed system 
will have an interoperability function allowing for the seamless transmission of name cards between PCs and mobile devices.

The rest of the paper is organized as follows: Section 2 provides an overview of the features of the $\mathrm{QR}$ code generator, the typesetting system, of name cards with and without QR codes, and of the mobile name card system. Section 3 describes the system architecture of PNCTS, which represents how to precisely calculate the type of text layout and how to operate the storage mechanism of text format. In Section 4 the prototype generation discusses the management and operation of PNCTS. Section 5 contains the experiment and discussion, and Section 6 provides a brief conclusion and future applications that would extend the present study.

\section{RELATED WORKS}

This section presents the recent status of name card typesetting systems with and without QR codes, and makes a brief review of previously published systems and methods.

\subsection{The $Q R$ code and code generator}

The QR code is a two-dimensional barcode [5] that can store at most 4,296 alphanumeric characters for devices that have an integrated camera, as in the case of most smart phones [6]. It requires the appropriate software to connect with web-based resources and provide related information for the product or service in question. QR codes can be scanned by mobile phones and, more importantly, are useful to many people. Wakahara et al. (2012) defines QR coding using the dotted picture multiplexing method and evaluates the redundancy characteristics. The QR code is made by the QR Editor, with dotted pictures that are scanned, decoded, and interpreted by the barcode reader of a mobile phone [7].

\subsection{The typesetting system}

Using typesetting software to generate name cards through a smart phone not only helps to give personal details without the necessity of having physical business cards in one's possession, but also provides for an almost unlimited number of customization possibilities in real-time. Earlier studies of computerized typesetting include a simple overview of the input area, edit functions, information retrieval, correction and make up, and computer programming. Data can be reformatted and composed for a phototypesetter automatically $[8,9]$. Subsequently, studies were done to determine methods to integrate non-textual elements such as special characters into the elements to be printed using a computerized typesetting system $[10,11]$. Then a typesetting application was created using PAGE-1 language [12]. Continuing to recent studies of computerized typesetting, a series of typesetting commands was developed for TEX documents [13], while typesets such as the Content Management System (CMS) used LATEX typesetting software as a developing tool. Moreover, some system applications were also developed for practical use, such as the LISP macros in the Emacs text editor [14]. This type of system is not limited by its typesetting environment. The implementation of a music processing system that can handle typesetting music, textual descriptions of music pieces and products, voices, etc. was examined [15]. A new method that extracts data from normal documents and transforms them into XML documents with automatic typesetting was presented [17], while a few years earlier another study developed an intellectual automatic typesetting system (IATS) to solve the bin packing problems [18]. Although typesetting software has existed for many years, the mobile devices have only recently been widely applied through apps.

\subsection{The traditional name card systems without $Q R$ code}

Most traditional name card software is applied to individual environments. Juicy Business Cards SWGSoft Inc. JuicyBC is a business card design software that can preview some business card templates created with JuicyBC through its web page. It is possible to design one's own unique business cards in as quickly as a few minutes, with access to quality background images and layouts. Cards can also be printed directly from one's own computer.

The business card software, LaughingBird Software, creates matching business cards to mix, match, and modify professionally designed letterhead and business card designs. This software includes 95 matching business card templates and letterhead designs.

BusinessCards MX 4.5 of Mojosoft Software helps to design and print a professional business card. The design process is based on ready-to-use elements to be accessible in the program, such as images and backgrounds, while freely shifting them on the prepared product. It uses ready-made templates or, if the individual or company is creatively inclined, independent designs.

In a separate study, a practical business card application model was created using a support system. The system manages aesthetic rules in an XML database and determines the business card layout by using a genetic algorithm. A facility allowing the user to edit the layout interactively is also provided. Because this is an interactive system, it takes time to finish a business card [19].

\subsection{The traditional name card system with $Q R$ code}

QR4 Europe, Netherlands, talks about the reason for including a QR code on a business card. It is more convenient to transfer contact information into the recipient's address book on the mobile phone through scanning. The alternative is retyping everything manually or using an OCR scanner. In many situations, these black-and-white blocks are not aesthetically appealing and do not complement the representation of a company as a business card should. After this system generates the QR code, it will be combined with a new name card according to the user's preferences. WebDesigner Depot has incorporated similar functions with QR4 Europe.

\subsection{Mobile name card system}

WorldCard Mobile, the first business card scanning application for Android, instantly transfers information from business cards to the user's native contacts. With a simple click of the camera, you no longer have to manually input contact information from business cards. Available in app stores, WorldCard Mobile has been adopted by over 20 million professionals.

CamCard is an Android-based application. It can capture business card images with a phone-mounted camera and recognize the card image content. The recognized result is automatically organized as a contact and saved into the phone's address book. At the time of writing CamCard had 
already been downloaded by more than 30 million people worldwide.

\section{SYSTEM ARCHITECTURE}

The system flow of the name card typesetting system is presented in Figure 1. The data collection from clients, system authentication, text typesetting, and QR code generation will be explained in the following sections.

\subsection{Data collection}

To generate a name card in advance, it is necessary to input seven texts into the textbox and select a QR code size, a QR code frame, a template, and a QR code color. Seven text items are required for input: user name, company's name, company's address, phone number, title, mobile number, and email address. The security functions of the encoder and decoder can be optionally applied to address and mobile phone number.

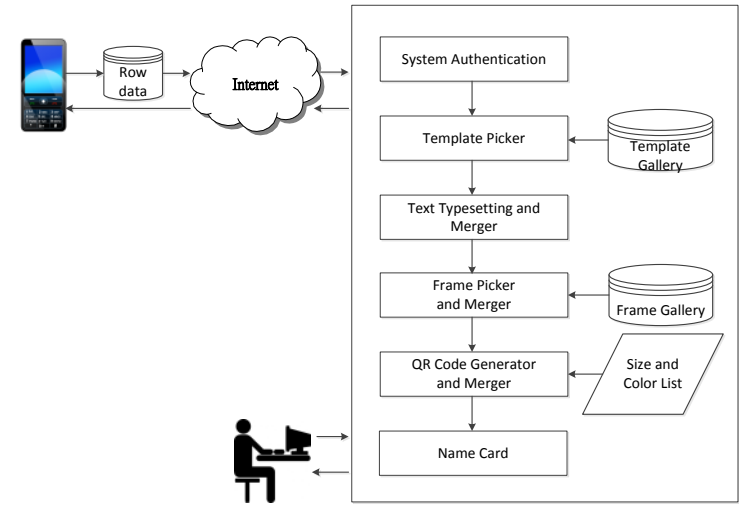

Fig 1: System flow

\subsection{System authentication}

This section discusses the authentication of the client's data transmission. In a previous study, the authentication mechanism was done using the HTTP web on the client side. It focuses on two scenarios: namely, the acknowledgment or rejection of the authentication process [20]. In the current study the HTTP web was considered on the server side, and the authentication mechanism suggested by Chung (2012) was reconfigured to match the current requirement. Figure 2 shows authentication success and the type of name card from the target server. When receiving name card requests from an unknown client (smart phone, tablet PC, etc.) authentication is an especially important aspect of picture file transmission. After authentication is approved by the picture server, the requested picture will be sent to the mobile device. The sequence charts of Figure 2 and Figure 3 present the user authentication phase, which is required from the mobile device. This system goes through HTTP protocol that sends data from client to server. Steps (1) and (2) request name card and data to be filled in on the web page. After a request is sent, step (3) HTTP parser requests the picture and sends a text message. Step (4a) requests picture for web server and then name card manager saves text messages through step (4b). If authentication on the web server is successful, steps (5), (6), and (7) will be executed. The left side of step (6) can save text messages and picture into storage. The right side of step (6) will send picture back to web server. Both activities happen at the same moment. Next, step (8) transmits the picture to the network manager. The picture manager receives picture data, sends metadata to a data manager, and outputs the picture to the mobile device's screen for viewing. Subsequently, the data manager collects field message and picture metadata through steps (9b) and (10a). Finally, step (11) sends the field of metadata to an event manager.

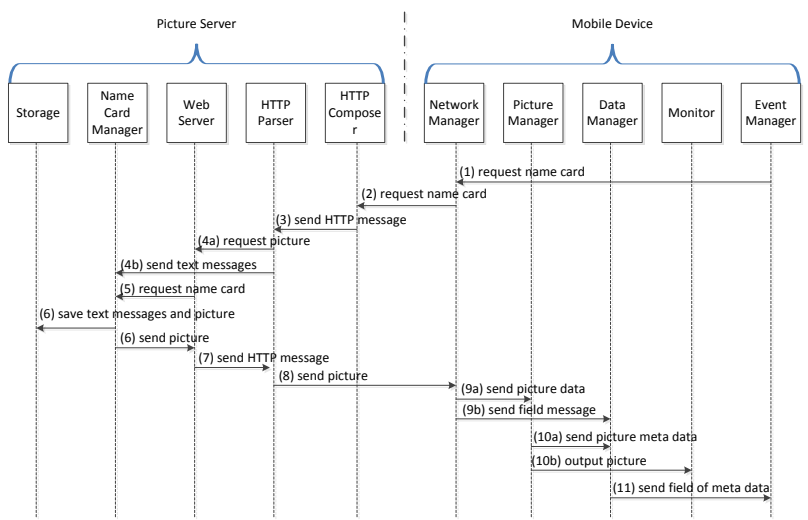

Fig 2: Sequence chart for authentication success

Another scenario is when the user authentication fails. When this situation happens, it can disrupt the normal procedure. As shown in Figure 3, HTTP parser passes two messages, steps $4 \mathrm{a}$ and $4 \mathrm{~b}$. Step (4a) requests picture for web server before saving text messages in step (4b). If authentication fails, the incident is documented by a web server, which deletes the text message on the name card manager automatically. Steps (5a) and (5b) occur simultaneously. After that, steps (6) and (7) send notice of authentication failure to the network manager and data manager. Finally, the event manager is informed via message about the authentication failure in step (8). Step (9) goes back to request the name card again. That authentication information includes identifier and password from the mobile device of login. Most likely, the app in the smart phone can help this work.

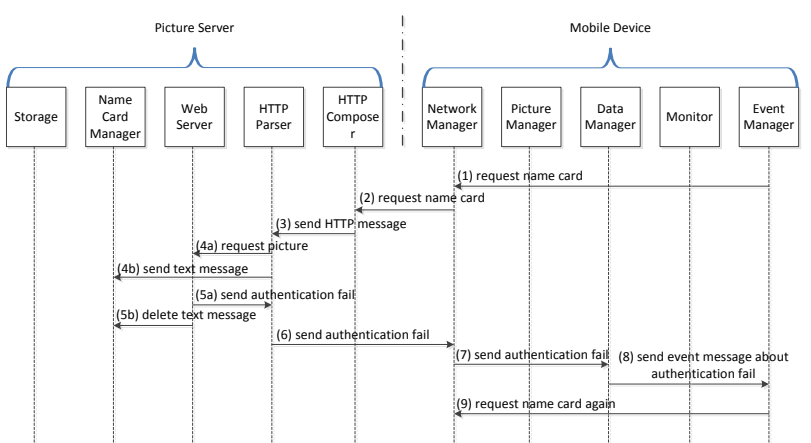

Fig 3: The sequence chart for authentication fails

\subsection{Typesetting method}

To calculate the types of text layout is an important work for typesetting. In order to conform the different text layouts to the opinions of users, a generating formula is set for four specific text layouts followed by Figure 4(a)(b)(c)(d). This work designated (a) as type 1, (b) as type 2 , (c) as type 3 , and (d) as type 4 . Lemma 1 represents the formula for the input point of each block. $d$ is distance, or a dynamic number between each block. The must be less than the minimum width and height of card. The variable "cardlength" is the length of card. The length of $\mathrm{T} 1, \mathrm{~T} 2$, and $\mathrm{T} 3$ are set as $\mathrm{W}$. The $\mathrm{k}$ can be adjusted for flexibility. In this work, $\mathrm{k}$ is either $3 / 2$ or $2 / 3$. Both proportions can make the name card look better. 

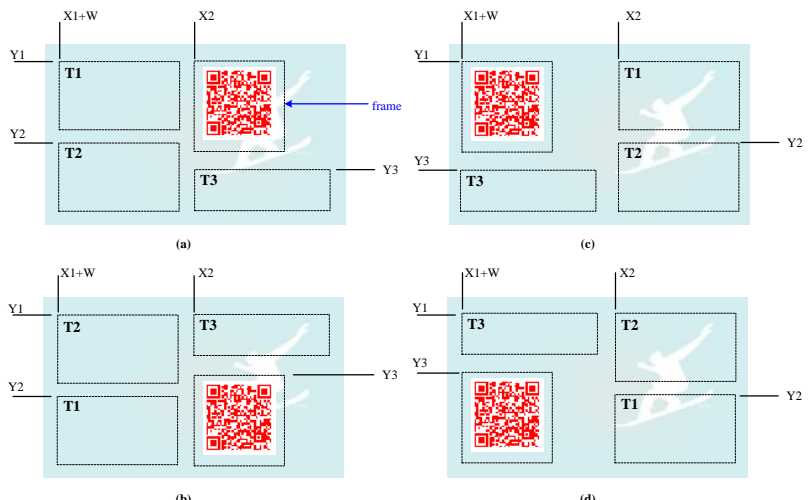

Fig 4: Types of text layout

Lemma 1

If given cardlength, $\mathrm{d}$, and $\mathrm{k} 1$ as the input, then all other parameters of name card are shown as below.

$\mathrm{W}=(1 / 2) \times$ cardlength $-\mathrm{d}$

$\mathrm{X} 2=(1 / 2) \times$ cardlength $+\mathrm{d}$

$\mathrm{Y} 2=(1 / 2) \times$ cardwidth $+\mathrm{d}$

$\mathrm{X} 1=\mathrm{d}$

$\mathrm{Y} 1=\mathrm{d}$

$\mathrm{Y} 3=\mathrm{k} \times \mathrm{Y} 2\left\{\begin{array}{l}\text { where } k=\frac{3}{2} \text { if text layout is type } 1 \text { and } 3 \\ \text { where } k=\frac{2}{3} \text { if text layout is type } 2 \text { and } 4\end{array}\right.$

Next, each text block includes a few text lines, as shown in Figure 5. The summary of Lemma 2 implies different text lines and locations. Based on different types, it can calculate different text blocks and the number of text lines of each block. T2 as an example is showing the relationship between text lines. Name cards generally come in four sizes: $9 \times 5.5,9 \times 5.4,9 \times 5$, or $9 \times 4.5 \mathrm{~cm}$. The size used in the present study was $9 \times 5.4 \mathrm{~cm}$. The $\delta$ of general size for the name card is closer to a width 340 pixels and a height of 204 pixels. Frame size followed by QR code size is the same type of size: large, medium, and small. In order to avoid non-readings from the $\mathrm{QR}$ code source figure-scanner, QR codes cannot be too small. This is why this work sets a source figure with three fixed sizes. QR codes can also be adjusted for position. Eventually, both Lemma 1 and Lemma 2 are applied into induction equations, or Lemma 3.

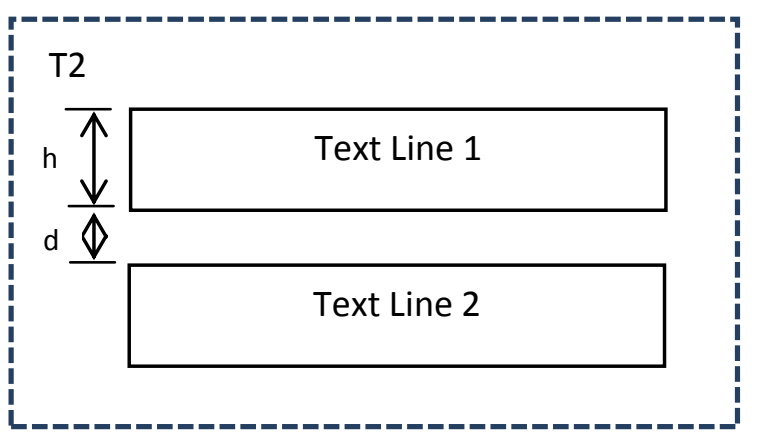

\section{Fig 5: The high and distance of text line}

\section{Lemma 2}

For each text line in a text block, as long as each di, hi, and number of types has been given by the client, the following formula is shown as the blocks of T1, T2, and T3.

T1:

$$
\begin{aligned}
& \mathrm{Y} 1+\sum_{\mathrm{i}=0}^{\mathrm{k}}(\mathrm{di}+\mathrm{hi}) \quad \text { type1 and } 3 \\
& \mathrm{Y} 2+\sum_{\mathrm{i}=0}^{\mathrm{k}}(\mathrm{di}+\mathrm{hi}) \quad \text { type } 2 \text { and } 4
\end{aligned}
$$

T2:

$$
\begin{aligned}
& \mathrm{Y} 2+\sum_{\mathrm{i}=0}^{\mathrm{k}}(\mathrm{di}+\mathrm{hi}) \quad \text { type } 1 \text { and } 3 \\
& \mathrm{Y} 1+\sum_{\mathrm{i}=0}^{\mathrm{k}}(\mathrm{di}+\mathrm{hi}) \quad \text { type } 2 \text { and } 4
\end{aligned}
$$

T3:

$$
\begin{aligned}
& \mathrm{Y} 3+\sum_{\mathrm{i}=0}^{\mathrm{k}}(\mathrm{di}+\mathrm{hi}) \quad \text { type1 and } 3 \\
& \mathrm{Y} 1+\sum_{\mathrm{i}=0}^{\mathrm{k}}(\mathrm{di}+\mathrm{hi}) \quad \text { type2 and } 4
\end{aligned}
$$

Hi: the height of font size

i: the number of text line

$\mathrm{d}$ : the distance of each text line

$\mathrm{k}$ : the total number of text line

$\mathrm{Tj}, \mathrm{Yj}:$ text area $\mathrm{j}, \mathrm{j}=1,2,3$

Lemma 3

The bound of name card should be understood and presented as follows.

$$
\begin{aligned}
& \mathrm{Y} 1+\sum_{\mathrm{i}=0}^{\mathrm{k}}(\mathrm{di}+\mathrm{hi})<=\mathrm{Y} 2 \\
& \mathrm{Y} 2+\sum_{\mathrm{i}=0}^{\mathrm{k}}(\mathrm{di}+\mathrm{hi})<=\delta \sim 204 \\
& \mathrm{Y} 3+\sum_{\mathrm{i}=0}^{\mathrm{k}}(\mathrm{di}+\mathrm{hi})<=\delta \sim 204
\end{aligned}
$$

Lemma 3 is organized from both Lemma 1 and Lemma 2. After the text strings are received, the XML file is available for layout storage that is easy for updating and querying. Many parameters are used to generate text stored into the XML file for each different text layout. Later, it will show a more detailed description. Then, every XML file can provide information that is convenient for Database through SQL to tracing history data. The schema of XML displays each parameter that locates text layout and text line. The meaning of $\mathrm{X} 1, \mathrm{X} 2, \mathrm{X} 3, \mathrm{Y} 1, \mathrm{Y} 2$, and $\mathrm{Y} 3$ is the distance for types of text layout. The purpose of the database is to record the index of each name card without creating extra variables and generating NULL data. 


\subsection{QR code generator and merger}

There are two works in the module of QR code generator and merger. First, even though Wakahara et al. (2012) provides their method to make the QR code with the dotted pictures, this paper reorganizes the flow chart of QR code generator in Figure 6. Two parameters, size and color, are selected by the client. All options are listed in the first sub-module. The base image is created as a background for the QR code. Base images are combinations of back image color and front image color. Colors are selected by the client. Next, pixel point fills the image file based on data set array, followed by the generated rule of QR code. After that, to create a targeted image, this prepares for both base image and targeted image being combined together. Finally, the combined image file has to be resized to a size determined by the client. This paper limits three sizes in QR code because of the consideration of name card size. Storage capacity for text is tremendous; otherwise, it is hard to typesetting when a large size of $\mathrm{QR}$ code is chosen.

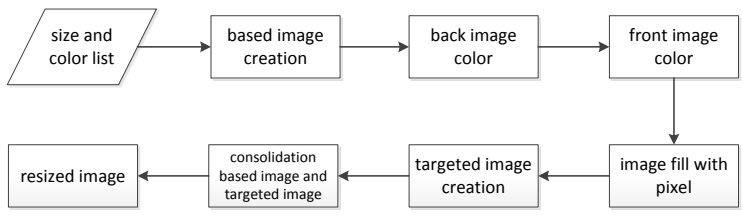

Fig 6: QR code generator and expansion flow

Last, this resized image file is merged into an existed semi-finished product. Here, the location of QR code with its frame follows the type of text layout that is set from the client in advance.

\section{PROTOTYPE GENERATION}

The prototype generated by the PNCTS is generated as shown in Figure 7.

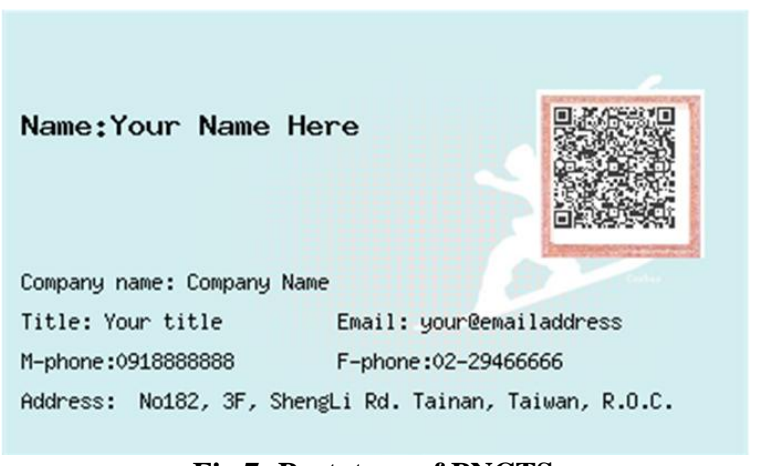

Fig 7: Prototype of PNCTS

Some management functions are listed as "preview", "save", "query all", "specific query", "update", "delete", and "exit". The "preview" button can send all input text to server side. And then, retrieving name card from processing is returned by the server. When the "save" button is pressed, the previewed name card can be saved onto mobile. Also, the total number of name cards is able to be counted and shown on the monitor. Any stored name cards can arbitrarily be updated and/or deleted by the client at any time. The control buttons are "update" and "delete". This paper proposes two query functions for management. One is "query all" which will retrieve all generated name cards in the mobile device. The client can review those name cards one by one on his or her mobile device. The other function is "specific query," which mentions an input string - either family name, first name, or other substring from seven input items. The client can get the related name cards that contain at least one substring. For example, the client types "Alex" and chooses one option of combo box "specific query". Then, all name cards in mobile phones, which have the same string between each sentence, should be caught up and displayed on the monitor immediately. That means the command "Alex" might result in more than one person being called.

\section{EXPERIMENT RESULTS}

The server uses an Apache HTTP server as its engine. The web browser is implemented using PHP, and is used to communicate with either a mobile device or a PC. The HTC Desire was used as the mobile test platform. The hardware specification is $1 \mathrm{GHz}$ CPU, $768 \mathrm{MB}$ RAM, and uses the Android 2.1 software. Simulation was implemented in an Android platform on Eclipse IDE using Java 6.0. The default setting of the environment is shown in Table 1.

Table 1. The meaning of symbols and their settings

\begin{tabular}{|c|c|c|c|}
\hline Symbol & Meanings & Domain & Default \\
\hline $\mathrm{M}$ & Name of input field & $3,4,5,6,7$ & 7 \\
\hline $\mathrm{Nc}$ & $\begin{array}{c}\text { Number of } \\
\text { characters }\end{array}$ & $30,60,90,120$ & 120 \\
\hline
\end{tabular}

\subsection{Query time of a name card, as compared to manual operation and PNCTS}

As shown in Table 2, experiments on the use of manual query a to find a name card from a set of 50, 100, 200, 500, and 1000 name cards showed significant time savings. Data in Table 2 is the mean query time after running the test three times consecutively.

Table 2. Query time for one name card under different scenarios

\begin{tabular}{|l|l|l|l|l|}
\hline $\begin{array}{l}\text { Num-b } \\
\text { er of } \\
\text { name } \\
\text { cards }\end{array}$ & $\begin{array}{l}\text { Manual } \\
(\mathbf{s e c})\end{array}$ & $\begin{array}{l}\text { Mobile } \\
\text { semi-auto } \\
\text { matic } \\
\text { (sec) }\end{array}$ & $\begin{array}{l}\text { Mobile } \\
\text { automatic } \\
\text { (sec) }\end{array}$ & $\begin{array}{l}\text { PC } \\
\text { automatic } \\
\text { (sec) }\end{array}$ \\
\hline 50 & 35 & 25 & 1 & $<<1$ \\
\hline 100 & 50 & 47 & 1 & $<<1$ \\
\hline 200 & 80 & 91 & 2 & $<<1$ \\
\hline 500 & 170 & 223 & 3 & $<<1$ \\
\hline 1000 & 320 & 443 & 4 & $<<1$ \\
\hline
\end{tabular}

Table 2 shows the query time of the four methods. (1) "Manual" means a specific name card is found by hand. (2)

"Mobile semi-automatic" means browsing name cards by switching windows. (3) When querying a specific name card on a mobile device the user is required to input the keyword or field. This method is called by "mobile automatic." (4) If PNCTS queries a specific name card through a PC, it is designated "PC automatic." The unit of query time is second. The " $<<$ " means the query time of 
PC is far lower than one second. When the number of name cards is over 100 , mobile semi-automatic takes more time than manual query. Because semi-automatic query works on a sliding window, it takes time to recognize a name card when switching windows. Mobile semi-automatic is suitable for browsing a small number of name cards but egregiously inefficient for larger quantities.

\subsection{PNCTS name card acquisition times across multiple platforms}

There are three phases: the typing phase, the selecting phase, and the card-generating phase. The typing phase includes typing records into the database. The choice of attributes belongs to the selecting phase. And the card-generating phase points to the time required to generate a name card, including pressing the button and waiting for a response from the system. The summary of the processing times is presented in Figure 8, which contains the averages of thirty trials. For the total time, the operating time of the mobile device was almost twice of that of the PC because of the size of keyboard. The key difference is that the size of the mobile device's keyboard is much smaller than that of the PC.

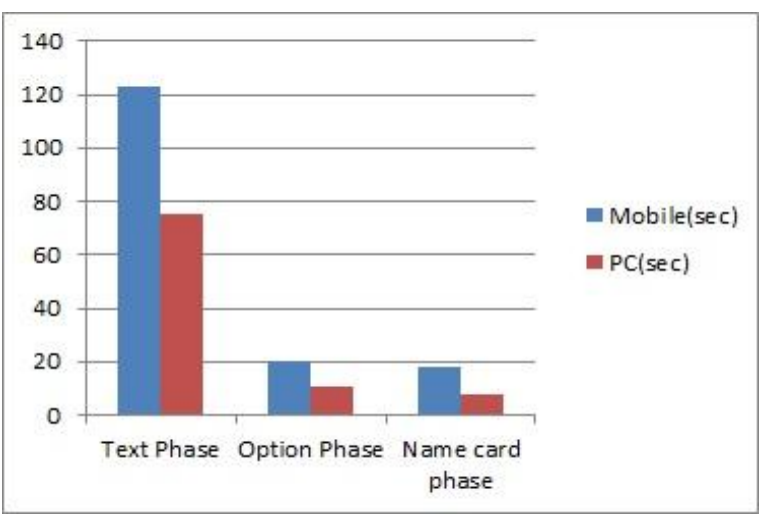

Fig 8: Graphical representation of the operating time between the two platforms

From Figure 8, it is apparent that the typing phase takes more time than either the selecting or card-generating phases. Specifically, time is directly proportional to the number of characters during the typing phase.

\subsection{Effect of the number of variables between mobile device and PC}

The number of variables varies from 3 to 7 types in each operation. Figure 9 represents the comparison between the two platforms. Both mobile device and PC took more time on the recognition and typing.

Each of the symbols contains a different description of specific attributes in order to conveniently remember and compare during the experiments, as shown in Table 3. For example, the user types text in $\mu 1$, including Name, Company, and Address fields. The system was tested using 130 characters, and it took an average of 142 seconds over 30 trials using the mobile device. Accordingly, typing text through PC only took 55 seconds.
Table 3. Description of Symbols

\begin{tabular}{|c|c|}
\hline Symbol & Description \\
\hline$\mu 1$ & Name, Company, Address \\
\hline$\mu 2$ & Name, Company, Address, Phone \\
\hline$\mu 3$ & Name, Company, Address, Phone, Title \\
\hline$\mu 4$ & Name, Company, Address, Phone, Title, \\
& Mobile number \\
\hline$\mu 5$ & $\begin{array}{r}\text { Name, Company, Address, Phone, Title, } \\
\text { Mobile number, Email address }\end{array}$ \\
\hline
\end{tabular}

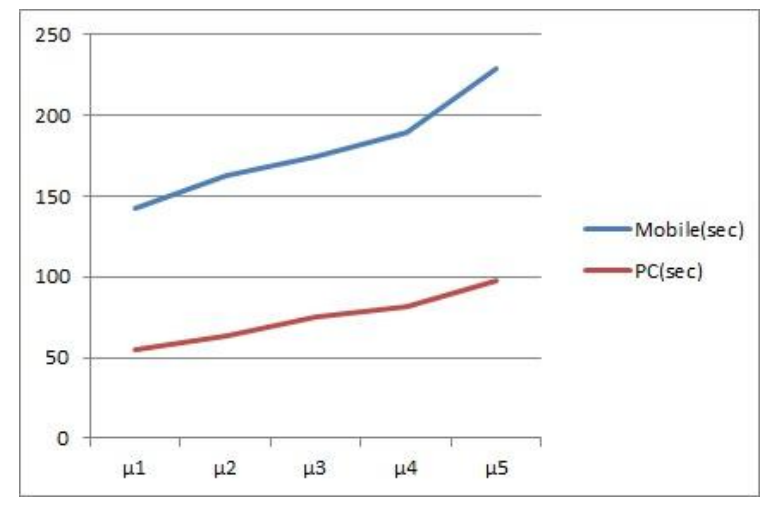

Fig 9: The comparison of attributes between mobile and PC

In Figure 9, as the number of attributes increases, the time cost is higher. Not surprisingly, a PC takes less time than a mobile device. From the result, it can be observed by two things. First, the percentage difference between the mobile device and PC in each symbol is 61.3, 61.3, 56.9, 56.8, and 57.2 , respectively. The maximum percentage difference in Table 3 is between symbols $\mu 1$ and $\mu 2$ and the minimum percentage difference in Table 3 is symbol $\mu 4$. Second, the range of the mobile device is 87 . And, the range of the PC is 43. The range variation of the symbol in the mobile device is higher than PC. That means a good input tool can affect the input performance of system, not terminal's business.

\subsection{Effect of number of characters}

The number of characters typed differs from 30, 60, 90, and 120 characters. Each of the symbols maps a fixed amount of characters this paper called for the experiments as $\mathrm{Nc} 1, \mathrm{Nc} 2, \mathrm{Nc} 3$, and $\mathrm{Nc} 4$. For instance the user types 30 characters in $\mathrm{Nc1}$ and it took an average of 81 seconds for 30 trials using the mobile device, while typing the same text through PC only required 20 seconds.

Figure 10 shows the typing time between mobile and PC The percentage difference between mobile device and PC in each symbol is $75.3,60.9,60.3$, and 56.8 , respectively. The symbol with maximum percentage difference among the symbols is $\mathrm{Nc1}$. Also, the minimum percentage 
difference of symbol is Nc4. Second, the range of values on the mobile device is 148 . The range of the PC is 79 . The range of variation of the symbols in the mobile is almost twice that of the PC.

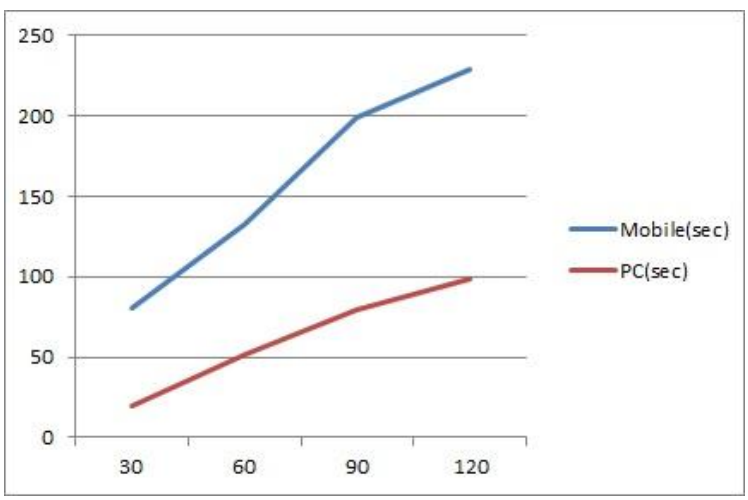

Fig 10: The comparison of characters between mobile and PC

In Figure 10, the trend of the chart is similar to the trend in Figure 9. Then those figures can help everyone to understand the attributes and characters are presented in a directly proportional relationship. In other words, a good typing environment for users can improve the input performance of mobile devices.

\section{CONCLUSIONS AND FUTURE WORKS}

This paper describes the design and implementation of a name card typesetting system in a mobile environment. It not only makes name cards, but also manages them conveniently. The PNCTS assists people who manage personal name cards and helps to keep their relationships organized. Also, people can share name cards with their specific friends through mobile devices in this system.

PNCTS is a personal digital name card system that features template, frame, size of $\mathrm{QR}$ code, and color of QR code customization options. Flexible layout templates and frames are available to users, who can set a preferred size and color for their QR code. PNCTS is portable and modifiable because it is a mobile device application. The $100 \%$ recognition ensures that important records will not be overlooked due to human error. Also, PNCTS can be more easily managed than other electronic name card systems, especially with respect to querying name cards and dialing out from the same mobile device. Lastly, PNCTS can be used from both mobile devices and computers.

From the results of the experiment, mobile semi-automatic takes more time than manual query after one hundred name cards. The application interface helps to manage name cards simply after advance generation. PNCTS automatic queries in both mobile and server side can query specific name cards based on conditional parameters. Typing on a mobile device is more time-consuming than doing so on a PC because of the inherently smaller keyboard size.

In this paper, it limits the text size and length of text lines because of the better layout to display on name card. Therefore, dynamic adjustment for font size, rows of text, and arrangement of text could add a new dimension as far as user customization is concerned. In the future, the authors will find a way to train users and reduce the chance of missing any data in this system due to improper operation. Another future plan is to replace manual typing with voice typing. However, both things should be investigated further before being implemented in future work. Lastly, the service can provide a mechanism for mobile payment. For instance, if the server supported new templates or frames for users, the latter would be able to pay for what he wants personally and control text layout on the template of his name card arbitrarily. Clearly, a wide range of applications of this research remains to be examined.

\section{ACKNOWLEDGMENTS}

Our thanks to experts were supported by NSC under the Grant NSC 100-2221-E-006-250-MY3.

\section{REFERENCES}

[1] Yue, L., Y. Ju, and L. Mingjun. Recognition of QR Code with Mobile Phones. in Control and Decision Conference, 2008. CCDC 2008. Chinese. 2008.

[2] Yu-Hsuan, C., C. Chung-Hua, and C. Ming-Syan. A General Scheme for Extracting QR Code from a Non-uniform Background in Camera Phones and Applications. in Multimedia, 2007. ISM 2007. Ninth IEEE International Symposium on. 2007.

[3] Yuan-Cheng, L., et al. A GPS Navigation System with QR Code Decoding and Friend Positioning in Smart Phones. in Education Technology and Computer (ICETC), 2010 2nd International Conference on. 2010.

[4] Sun, A., Y. Sun, and C. Liu, The QR-code Reorganization in Illegible Snapshots Taken by Mobile Phones, in Proceedings of the International Conference Computational Science and its Applications. 2007, IEEE Computer Society. p. 532-538.

[5] Sutheebanjard, P. and W. Premchaiswadi. QR-code Generator. in Knowledge Engineering, 2010 8th International Conference on ICT and. 2010.

[6] Linden, G., M. Conover, and J. Robertson, The Netflix Prize, Computer Science Outreach, and Japanese Mobile Phones. Commun. ACM, 2009. 52(10): p. 8-9.

[7] Wakahara, T., N. Yamamoto, and H. Ochi, Image Processing of Dotted Picture in QR Code of Cellular Phones. Computer Systems Science and Engineering, 2012. 27(1): p. 73-81.

[8] Boyle, J.J., Automated Typesetting of Existing Computer Tabular Data. IEEE Transactions on Engineering Writing and Speech, 1968. EW11(2): p. 129-\&.

[9] Boyle, J.J., Computerized Typesetting - An Overview. IEEE Transactions on Engineering Writing and Speech, 1969. EW12(2): p. 28-\&.

[10] Kuney, J.H., Processing Manuscripts for Input to A Computerized Typesetting System for Scientific Journals. IEEE Transactions on Engineering Writing and Speech, 1969. EW12(2): p. 49-\&.

[11] Corley, F.C., High-Quality Computer Typesetting for Text, Formulas, and Listings. IEEE Transactions on Engineering Writing and Speech, 1969. EW12(2): p. 46-\&. 
[12] Pierson, J.L., Computer Program for Electronic Typesetting. IEEE Transactions on Engineering Writing and Speech, 1971. EW14(2): p. 46-52.

[13] Iwasaki, H., Developing a Lisp-based Preprocessor for TEX Documents. Software-Practice \& Experience, 2002. 32(14): p. 1345-1363.

[14] Fernandez-Iglesias, M.J., et al., Generating High Quality Printouts from Content Management Systems: A Cost-Effective Approach. Software-Practice \& Experience, 2005. 35(11): p. 1007-1026.

[15] Filgueiras, M., Implementing a Symbolic Music Processing System. Software-Practice \& Experience, 1998. 28(5): p. 493-512.

[16] De Vivo, C. and C. Metelli, On The Typeset of A Butler B(2)-GROUP. Houston Journal of Mathematics, 2012. 38(3): p. 653-683.
[17] Yang, Q.C. and J.F. Zhu, Technology of Document Automatic Typesetting Based on XML. 2012 2nd International Conference on Applied Social Science, ed. J. Hu. 2012, Newark: Information Engineering Research Inst, USA. 354-360.

[18] Lee, S.L., A Decision Support System for Luggage Typesetting. Expert Systems with Applications, 2008. 35(4): p. 1620-1627.

[19] Harada, S., K. Morinaga, and M. Hirakawa, Development of an Interactive Business Card Creation Support System Using Genetic Algorithm. Proceedings of the 2005 International Conference on Active Media Technology, ed. H. Tarumi, Y. Li, and T. Yoshida. 2005. 85-88

[20] Chung, W.-H., A Smartphone Watch for Mobile Surveillance Service. Personal and Ubiquitous Computing, 2012. 16(6): p. 687-696. 